\title{
Response of Sport Horses to Different Formulations of Equine Influenza Vaccine
}

\author{
Johanna Entenfellner ${ }^{1}$, Jacinta Gahan ${ }^{2}$, Marie Garvey ${ }^{2}$, Cathal Walsh ${ }^{3}{ }^{\circledR}$, Monica Venner ${ }^{4}$ \\ and Ann Cullinane ${ }^{2, *}$ \\ 1 School of Veterinary Medicine, Bischofsholer Damm 15, 30173 Hannover, Germany; \\ johannaentenfellner@icloud.com \\ 2 Irish Equine Centre, Johnstown, Naas, Co. Kildare, W91 RH93 Johnstown, Ireland; \\ JGahan@irishequinecentre.ie (J.G.); MGarvey@irishequinecentre.ie (M.G.) \\ 3 Department of Mathematics and Statistics, University of Limerick, V94 T9PX Limerick, Ireland; \\ Cathal.Walsh@ul.ie \\ 4 Pferdeklinik Destedt GmbH, Destedt, Trift 4, 38162 Cremlingen, Germany; MVenner@gmx.de \\ * Correspondence: ACullinane@irishequinecentre.ie; Tel.: +353-45-866-266
}

Received: 12 June 2020; Accepted: 7 July 2020; Published: 10 July 2020

\begin{abstract}
The international governing body of equestrian sports requires that horses be vaccinated against equine influenza within 6 months and 21 days of competing. The aim of this study was to compare the antibody response of young sport horses to six-monthly booster vaccination with equine influenza vaccines of different formulations. An inactivated vaccine was allocated to 35 horses and subunit and recombinant vaccines were allocated to 34 horses each. After vaccination, all horses were monitored for evidence of adverse reactions. Whole blood samples were collected at the time of vaccination and on nine occasions up to six months and 21 days post vaccination. Antibodies against equine influenza were measured by single radial haemolysis. Transient fever and injection site reactions were observed in several horses vaccinated with each vaccine. Only two horses failed to seroconvert post booster vaccination but there was a delayed response to the recombinant vaccine. The antibody response to the recombinant vaccine was lower than that induced by the whole-inactivated and subunit vaccines up to three months post vaccination. Thereafter, there was no significant difference. By six months post vaccination, the majority of horses in all three groups were clinically but not virologically protected. There was minimal decline in antibody titres within the 21-day grace period.
\end{abstract}

Keywords: equine influenza; inactivated; subunit; recombinant vaccines; sport horse; antibody; reactogenicity

\section{Introduction}

The economic impact of equine influenza is minimised by vaccination with vaccines that decrease virus shedding and clinical signs [1,2]. However, the protection induced by vaccination is short lived and multiple doses of vaccine are required to protect highly mobile horses and safeguard equestrian events [3,4]. The vaccines that are most widely available globally are of three types: whole inactivated, subunit and recombinant [2]. The objective of this study was to compare the humoral immune response after booster vaccination with a whole-inactivated vaccine (Duvaxyn IE-T Plus), an immune-stimulating complex (ISCOM)-based subunit vaccine (EquipFT) and a canarypox recombinant vaccine (Proteq Flu-Te). Antibodies against the envelope glycoprotein haemagglutinin (HA) have been demonstrated to protect against equine influenza both in experimental challenge studies and in outbreaks in the field [5-9]. These antibodies can be measured by the haemagglutination 
inhibition (HI) test or the single radial haemolysis (SRH) test but only the SRH test is standardised internationally with reference sera available from the European Directorate for the Quality of Medicines (EDQM) [10,11]. SRH antibody titres are established correlates of protection. If the vaccine strain is closely related to the challenge virus, antibody titres $\geq 150 \mathrm{~mm}^{2}$ are associated with protection against infection commonly referred to as virological protection, and antibody titres $\geq 85 \mathrm{~mm}^{2}$ are associated with clinical protection but horses may shed virus $[5,6,12]$. Thus, evaluation of SRH antibody titres is accepted as an evidence-based approach to the examination of vaccine efficacy in the field where experimental virus challenge is not feasible. The majority of virus challenge studies are limited to primary vaccination consisting of two or three doses of vaccine and rarely shed any light on the status of horses that have been regularly vaccinated [2]. In the past, we have compared the response of Thoroughbred weanlings and National Hunt racehorses to primary vaccination and annual booster vaccination, respectively, with inactivated, subunit and recombinant vaccines $[13,14]$. In this study, we compared the response of young sport horses to six-monthly booster vaccination with similar vaccines.

\section{Materials and Methods}

\subsection{Horses}

This study was undertaken on a single farm to compare the humoral response to booster vaccination with three different EI vaccines. One hundred and three horses were available for vaccination. Horses were chosen sequentially for vaccination with the same product, with 35 in the first group and 34 in the other groups, with the order of vaccines chosen at random. Animals were followed up longitudinally with repeated samples drawn at ten time points. After approval by the Ethics Committee of the Veterinary School of Hannover, the study was registered by the authorities of the Meclenburg-Vorpommern. The horses ranged in age from 2 to 7 years old (median: 3 years) at the time of vaccination when, on average, it was $172( \pm 11.4)$ days since their last vaccination. The horses had received from 4 to 15 (mean $6.5 \pm 2.2$ ) doses of vaccine prior to this study.

\subsection{Vaccines}

A whole-inactivated vaccine Duvaxyn IE-T Plus (Elanco Animal Health, Eli Lilly Ltd., Basingstoke, United Kingdom), an ISCOM subunit vaccine EquipFT (Zoetis, Parsippany-Troy Hills, NJ, USA) and a recombinant canarypox vaccine ProteqFlu-Te (Merial S.A.S./Boehringer Ingelheim, 29 Avenue Tony Garnier, 69007, Lyon, France) were all purchased commercially for inclusion in this study. The different adjuvants and composition of each of the three vaccines are shown in Table 1.

Table 1. Vaccine product details.

\begin{tabular}{|c|c|c|c|c|}
\hline $\begin{array}{l}\text { Vaccine } \\
\text { Producer }\end{array}$ & Nature & Adjuvant & Virus Strains & $\begin{array}{l}\text { Tetanus } \\
\text { Toxoid }\end{array}$ \\
\hline Duvaxyn IE-T Plus (Elanco) ${ }^{\circledR}$ & Inactivated whole virus & $\begin{array}{l}\text { Carbomer } \\
\text { Aluminium hydroxide }\end{array}$ & $\begin{array}{c}\text { A/eq/Prague/56 (H7N7) } \\
\text { A/eq/Suffolk/89 (H3N8) } \\
\text { A/eq/Newmarket/1/93 (H3N8) }\end{array}$ & >150 I.U. \\
\hline Equip FT (Zoetis) ${ }^{\circledR}$ & Subunit & $\begin{array}{l}\text { ISCOM } \\
\text { Quillaic acid derivative } \\
\text { Aluminium-phosphate }\end{array}$ & $\begin{array}{c}\text { A/eq/Newmarket/77 (H7N7) } \\
\text { A/eq/Kentucky/98 (H3N8) } \\
\text { A/eq/Borlange/91 (H3N8) }\end{array}$ & $100 \mathrm{Lf}$ \\
\hline ProteqFlu TE (Merial) ${ }^{\circledR}$ & Canarypox-recombinant & Carbomer & $\begin{array}{c}\text { A/eq/Ohio/03 (H3N8) } \\
\text { A/eq/Richmond/1/07 (H3N8) }\end{array}$ & $>30$ I.U. \\
\hline
\end{tabular}

ISCOM = immune-stimulating complexes, $\mathrm{IU}=$ international unit, and $\mathrm{Lf}=$ limes flocculation units.

\subsection{Vaccination}

The horses in this study were randomly allocated one of the three vaccines. The inactivated vaccine was allocated to 35 horses and the subunit and recombinant vaccines were allocated to 34 horses each. Gender was not a factor in vaccine allocation but mares, geldings and stallions were included in each group. Vaccines were administered by deep intra muscular injection which was performed by the 
attending veterinary surgeon. Following vaccination, all horses were observed for evidence of adverse local and/or systemic reactions. Rectal temperatures were taken daily for three days post vaccination.

\subsection{Collection of Samples}

Whole blood samples were collected by the attending veterinary surgeon on the day of vaccination (D0) and days seven (D7), 14 (D14), 21 (D21), 60 (D60), 90 (D90), 121 (D121), 150 (D150), 180 (D180) and 201 (D201) post booster vaccination. Due to animal handling limitations, some of the horses could not be sampled at one or more of the sampling times and horses were lost to the study at different times as they were sold. The number of horses sampled on each occasion is shown in Table 2.

Table 2. Number of horses sampled at each time point post vaccination.

\begin{tabular}{|c|c|c|c|c|c|c|c|c|c|c|}
\hline Vaccine & D0 & D7 & D14 & D21 & D60 & D90 & D121 & D150 & D180 & D201 \\
\hline Duvaxyn IET Plus & 35 & 34 & 32 & 34 & 29 & 30 & 30 & 23 & 23 & 20 \\
\hline Equip $\mathrm{FT}^{\circledR}$ & 34 & 33 & 34 & 33 & 26 & 26 & 26 & 20 & 20 & 15 \\
\hline Proteq Flu $\mathrm{Te}^{\circledR}$ & 34 & 34 & 31 & 30 & 23 & 23 & 23 & 18 & 18 & 14 \\
\hline Total & 103 & 101 & 97 & 97 & 78 & 79 & 79 & 61 & 61 & 49 \\
\hline
\end{tabular}

\subsection{Serology}

Antibodies against two H3N8 viruses A/equine/Meath/1/2007 (Florida Clade 2) and A/equine/South Africa/4/2003 (Florida Clade 1) were measured using the SRH test as previously described [15]. A/equine/Meath/1/07 demonstrates 100\% HA nucleotide identity to the Florida clade 2 World Organisation for Animal Health (OIE)-recommended vaccine strain A/equine /Richmond/1/07 [16] A/equine/South Africa/4/2003 is one of the OIE-recommended Florida clade 1 vaccine strains. The degree of HA amino acid identity between the strains used in the vaccines and the strains used to conduct SRH is presented in Table 3. The area of haemolysis resulting from the lysis of equine influenza antigen-coated SRBCs by the antibody in the test sera were expressed in $\mathrm{mm}^{2}$. Mean H3N8 antibody values, i.e., against A/equine/Meath/1/2007 and A/equine/South Africa/4/2003, were calculated from $\mathrm{SRH}$ results obtained and these are presented in Supplementary Table S1. A significant increase in antibody titre was defined as an increase of $\geq 25 \mathrm{~mm}^{2}$ [9]. Similarly, a significant decline in antibody titre was defined as a decrease of $\geq 25 \mathrm{~mm}^{2}$. The laboratory investigator was blind to vaccine allocation to individual horses. The response to tetanus toxoid following vaccination was not examined during this study.

Table 3. The degree of HA amino acid identity (\%) between the strains used in the vaccines and the strains used to conduct SRH, A/equine/Meath/1/2007 and A/equine/South Africa/4/2003.

\begin{tabular}{|c|c|c|c|c|}
\hline \multirow{2}{*}{ Vaccine } & \multirow[b]{2}{*}{ Nature } & \multirow{2}{*}{ Vaccine Virus Strains } & \multicolumn{2}{|c|}{ Amino Acid Identity (\%) } \\
\hline & & & A/eq/South Africa/4/03 & A/eq/Meath/1/07 \\
\hline \multirow{3}{*}{ Duvaxyn IE-T Plus (Elanco) ${ }^{\circledR}$} & \multirow{3}{*}{ Inactivated whole virus } & A/eq/Prague/56 (H7N7) & 47.69 & 47.51 \\
\hline & & A/eq/Suffolk/89 (H3N8) & 97.27 & 96.90 \\
\hline & & A/eq/Newmarket/1/93 (H3N8) & $96.96 *$ & $96.96 *$ \\
\hline \multirow{3}{*}{ Equip FT (Zoetis) ${ }^{\circledR}$} & \multirow{3}{*}{ Subunit } & A/eq/Newmarket/77(H7N7) & 47.51 & 47.34 \\
\hline & & A/eq/Kentucky/98 (H3N8) & $96.04 *$ & $96.04 *$ \\
\hline & & A/eq/Borlange/91 (H3N8) & $92.70 *$ & $92.40 *$ \\
\hline \multirow{2}{*}{ ProteqFlu TE (Merial) ${ }^{\circledR}$} & \multirow{2}{*}{ Canarypox-recombinant } & A/eq/Ohio/03 (H3N8) & 100 & 98.90 \\
\hline & & A/eq/Richmond/1/07 (H3N8) & 98.90 & 100 \\
\hline
\end{tabular}

\subsection{Statistical Analysis}

All statistical analysis was carried out on the open-source package R version 3.1.1 (CRAN, Dublin, Ireland) [17]. A significance level of $p<0.05$ was used for all statistical tests. Pearson's correlation coefficient was used to examine whether there was a correlation between age and number of vaccines received prior to the study. A Kruskal-Wallis test was used to examine the association between baseline titre and categorical variables such as gender and vaccine type. Linear models, using main effects only, 
were used to examine the association between continuous variables, such as age at first vaccination and baseline titre. Pearson's chi-squared test was used to determine if there was a significant difference between the vaccines in their association with pyrexia and local inflammation. Data were analysed using a repeated-measures analysis of variance and post hoc testing was carried out with Tukey's HSD. Residuals were examined visually for normality. The area under the curve (AUC) as described by Heldens et al. (2002) [18] was calculated by the trapezoidal rule and used as the metric for the repeated-measures analysis of antibody levels. To examine the change in titre between the final two time points in the study, a paired $t$-test was used for each vaccine.

\section{Results}

\subsection{Pre-Existing Immune Status}

The equine population in this study was investigated for factors that influenced baseline SRH titre. In univariate models, the greater the number of vaccinations previously received and the older the horse, the greater the baseline SRH titre on D0 $(p<0.001)$. However, these factors are confounding, as the older horses had received more vaccinations (Pearson's correlation co-efficient $=0.97)$. Gender $(p>0.9)$ or age at first vaccination $(p=0.8)$ did not significantly affect the baseline titre. The D0 baseline SRH titre was similar for the three vaccine groups $(p=0.1)$.

\subsection{Reaction to Vaccination}

Four horses mounted a febrile response post vaccination with the whole-inactivated vaccine. A transient increase in temperature was observed in two horses on day two (D2) $\left(38.5\right.$ and $\left.38.7^{\circ} \mathrm{C}\right)$ and in two horses on day 3 (D3) $\left(38.5\right.$ and $38.6^{\circ} \mathrm{C}$ ) post vaccination. Three horses developed (one on D2 and two on D3) a small local swelling at the injection site, two of which were painful on palpitation.

Five horses mounted a febrile response post vaccination with the subunit vaccine. A transient increase in temperature was observed in four horses on D2 $\left(38.5-38.7^{\circ} \mathrm{C}\right)$ and in one horse on D3 post vaccination $\left(38.5^{\circ} \mathrm{C}\right)$. Seven horses experienced transient injection site reactions three of which were painful on palpitation. Two of the horses with local reactions were pyrexic.

Seven horses mounted a febrile response post vaccination with the recombinant vaccine. An increase in temperature was observed in five horses on D2 $\left(38.6-38.9^{\circ} \mathrm{C}\right)$ and two horses on D3 (38.6 and 38.7). Two of the horses that spiked a temperature on D2 were still pyrexic $\left(39.5-38.7^{\circ} \mathrm{C}\right)$ on D3, and one of these developed an injection site reaction on D2. In total, nine horses developed injection site reactions, two of which were painful on palpitation.

There was no significant difference between the vaccine groups in the incidence of pyrexia $(p=0.6)$ or local inflammation $(p=0.1)$.

\subsection{Immune Response to Booster Vaccination}

The mean SRH values for the three vaccine groups at each sampling point are illustrated in Figure 1 and all the results for individual horses are presented in Supplementary Table S1. All of 33 horses vaccinated with the subunit vaccine and all but one of the horses vaccinated with the whole-inactivated vaccine seroconverted by D7. In contrast, none of the horses vaccinated with the canarypox vaccine seroconverted by D7. Twenty two of 31 horses vaccinated with the canarypox vaccine seroconverted between D7 and D14, and two of the remaining horses seroconverted between D14 and D21.

This study did not include experimental challenge but based on antibody titre on D21, all horses vaccinated with the whole-inactivated vaccine tested were expected to be virologically protected, as were $97 \%$ of those vaccinated with the ISCOM subunit vaccine but only $73 \%$ of the horses vaccinated with the canarypox recombinant vaccine. After D21, the SRH antibody titres decreased for all vaccine groups. By D60, although $61 \%$ of horses vaccinated with the whole-inactivated vaccine had experienced a significant decline $\left(\geq 25 \mathrm{~mm}^{2}\right), 93 \%$ of the horses sampled were virologically protected with titres $\geq 150 \mathrm{~mm}^{2}$. This compared favourably with $62 \%$ and $48 \%$ of the horses vaccinated with the 
subunit and canarypox vaccines, respectively. Titres continued to decline for all vaccine groups and by three months post vaccination (D90), only $50 \%$ of the horses vaccinated with the inactivated vaccine were expected to be virologically protected, as were $27 \%$ and $30 \%$ of those vaccinated with the subunit and canarypox vaccines, respectively. By six months post vaccination, with very few exceptions, the horses in all three groups were expected to be clinically but not virologically protected.

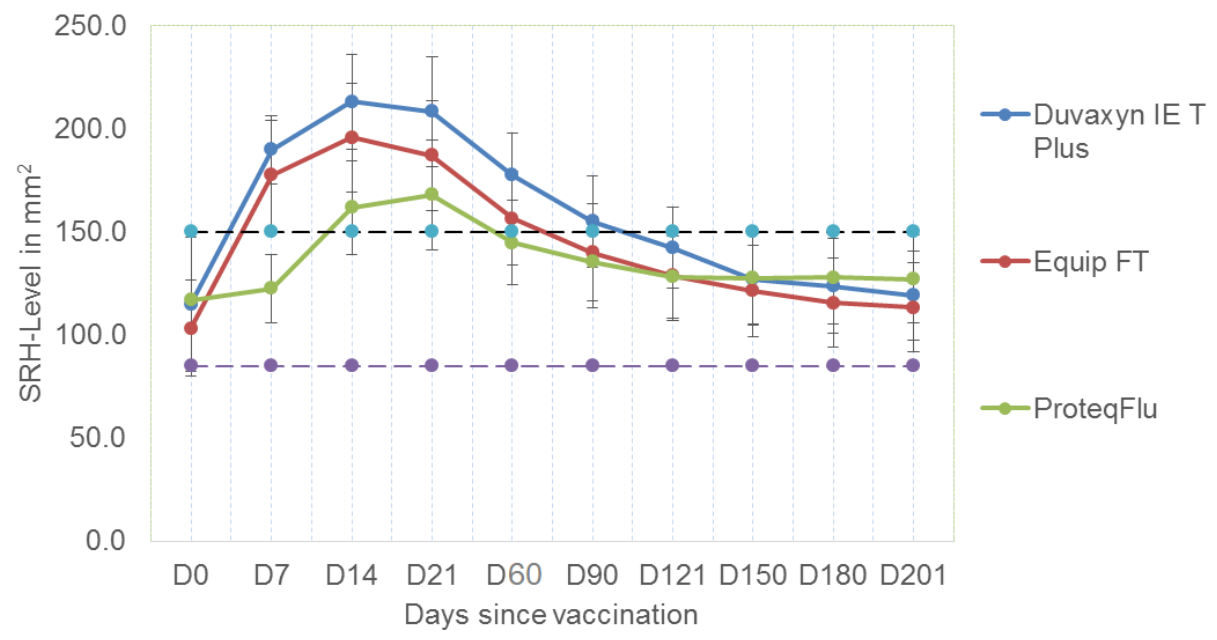

Figure 1. The mean SRH values with standard deviation bars for the three vaccine groups, those vaccinated with a whole-inactivated vaccine (Duvaxyn IE-T Plus), an ISCOM subunit vaccine (EquipFT) and a recombinant canarypox vaccine (ProteqFlu-Te), at each sampling point, i.e., the day of vaccination (D0) and days 7, 14, 21, 60, 90, 121, 150, 180 and 201 post vaccination. The black dashed line with blue circles indicates $150 \mathrm{~mm}^{2}$, the threshold level of SRH antibodies associated with virological protection. The purple dashed line indicates $85 \mathrm{~mm}^{2}$, the threshold level of SRH antibodies associated with clinical protection.

Tukey's HSD was carried out to examine significant differences in performance between the three vaccines included in this study. Results of this analysis are shown in Table 4 and include the comparison of all possible pairs of means, that is they apply simultaneously to the set of all pairwise comparisons. The antibody response of the horses vaccinated with the whole-inactivated and subunit vaccines was not significantly different at any time point but the response of both groups was significantly better than that elicited by vaccination with the canarypox recombinant vaccine up to 90 days post vaccination. At 121, 150, 180 and 201 days post vaccination, there was no significant difference between the antibody response of the three groups.

Table 4. Post hoc Tukey's HSD comparisons. Performance of the vaccines at different time points post booster vaccination compared using Tukey's HSD. Negative values indicate a greater change from baseline for the latter vaccine in each paired comparison.

\begin{tabular}{cccc}
\hline \multirow{2}{*}{ Time Points } & \multicolumn{2}{c}{$\mathbf{9 5 \% \text { Confidence Interval }}$} & \multirow{2}{*}{$\boldsymbol{*}$ Value } \\
\cline { 2 - 3 } & Lower & Upper & \\
\hline D0-D7 & & & \\
EquipFT-Duvaxyn IE-T Plus & -11.61 & 14.22 & $>0.9$ \\
ProteqFLU TE-Duvaxyn IE-T Plus & -81.04 & -55.4 & $<0.001^{*}$ \\
ProteqFLU TE-EquipFT & -82.44 & -56.61 & $<0.001^{*}$ \\
D0-D14 & & & \\
EquipFT-Duvaxyn IE-T Plus & -25.42 & 12.9 & 0.7 \\
ProteqFLU TE-Duvaxyn IE-T Plus & -72.83 & -33.6 & $<0.001^{*}$ \\
ProteqFLU TE-EquipFT & -66.3 & -27.7 & $<0.001^{*}$ \\
\hline
\end{tabular}


Table 4. Cont.

\begin{tabular}{|c|c|c|c|}
\hline \multirow{2}{*}{ Time Points } & \multicolumn{2}{|c|}{ 95\% Confidence Interval } & \multirow{2}{*}{$p$ Value } \\
\hline & Lower & Upper & \\
\hline \multicolumn{4}{|l|}{ D0-D21 } \\
\hline EquipFT-Duvaxyn IE-T Plus & -27.1 & 7.50 & 0.4 \\
\hline ProteqFLU TE-Duvaxyn IE-T Plus & -62.2 & -26.75 & $<0.001$ * \\
\hline ProteqFLU TE-EquipFT & -52.7 & -16.71 & $<0.001 *$ \\
\hline \multicolumn{4}{|l|}{ D0-D60 } \\
\hline EquipFT-Duvaxyn IE-T Plus & -24.73 & 11.16 & 0.7 \\
\hline ProteqFLU TE-Duvaxyn IE-T Plus & -54.48 & -17.38 & $<0.001 *$ \\
\hline ProteqFLU TE-EquipFT & -48.17 & -4.18 & $<0.01 *$ \\
\hline \multicolumn{4}{|l|}{ D0-D90 } \\
\hline EquipFT-Duvaxyn IE-T Plus & -18.59 & 14.72 & $>0.9$ \\
\hline ProteqFLU TE-Duvaxyn IE-T Plus & -41.14 & -6.69 & $<0.01 *$ \\
\hline ProteqFLU TE-EquipFT & -48.17 & -4.18 & $<0.01 *$ \\
\hline \multicolumn{4}{|l|}{ D0-D121 } \\
\hline EquipFT-Duvaxyn IE-T Plus & -15.55 & 14.69 & $>0.9$ \\
\hline ProteqFLU TE-Duvaxyn IE-T Plus & -34.14 & -2.86 & $0.016^{*}$ \\
\hline ProteqFLU TE-EquipFT & -34.22 & -1.9 & $0.02 *$ \\
\hline \multicolumn{4}{|l|}{ D0-D150 } \\
\hline EquipFT-Duvaxyn IE-T Plus & -4.98 & 24.16 & 0.3 \\
\hline ProteqFLU TE-Duvaxyn IE-T Plus & -18.04 & 12.27 & 0.9 \\
\hline ProteqFLU TE-EquipFT & -28.26 & 3.05 & 0.1 \\
\hline \multicolumn{4}{|l|}{ D0-D180 } \\
\hline EquipFT-Duvaxyn IE-T Plus & -7.3 & 21.71 & 0.5 \\
\hline ProteqFLU TE-Duvaxyn IE-T Plus & -14.03 & 15.84 & $>0.9$ \\
\hline ProteqFLU TE-EquipFT & -21.71 & 9.13 & 0.6 \\
\hline \multicolumn{4}{|l|}{ D0-D201 } \\
\hline EquipFT-Duvaxyn IE-T Plus & -7.95 & 25.05 & 0.4 \\
\hline ProteqFLU TE-Duvaxyn IE-T Plus & -14.2 & 19.5 & $>0.9$ \\
\hline ProteqFLU TE-EquipFT & -23.9 & 12.0 & 0.7 \\
\hline
\end{tabular}

* significant difference.

The area under the curve (AUC) against the SRH antigens tested was also calculated for each of the three vaccines. This was performed using complete case data $(n=52)$ up to day 180 , since missing data resulted in only 42 complete cases at day 201. The magnitude of the immune response measured by AUC analysis revealed a significantly lower antibody response for the subunit and canarypox vaccines compared to the whole-inactivated vaccine. There was a significant difference between the AUC for the subunit and whole-inactivated vaccine $(p=0.046)$, and the canarypox recombinant vaccine and whole-inactivated vaccine $(p=0.008)$ but not between the subunit and canarypox recombinant vaccine $(p=0.67)$.

A question of interest is the change in titre during the FEI grace period, from day 180 to day 201. For each vaccine, this change was examined using a paired $t$-test. For Duvaxyn IE-T Plus, there was a decline of $3.79 \mathrm{~mm}^{2}$ (95\% CI 2.0 to 5.6); for EquipFT, there was a decline of $2.8 \mathrm{~mm}^{2}$ (95\% CI 0.6 to 5.0); and for ProteqFlu TE, there was no statistically significant change-a decline of $0.02 \mathrm{~mm}^{2}(95 \%$ CI 2.8 to -2.8$)$.

\section{Discussion}

This study compared the antibody responses to commercial equine influenza vaccines for six months following a biannual booster. A subset of the serology results were previously included in a global project to establish within the context of existing OIE standards, a science-based rationale to identify the ideal time period for equine influenza vaccination prior to shipment [19]. The current study focused on a comparison of vaccine performance including adverse effects in a population consisting of young regularly vaccinated sport horses. At the time of vaccination, their mean SRH antibody titre of $112 \mathrm{~mm}^{2}$ was slightly lower than that of $123 \mathrm{~mm}^{2}$ recorded previously for horses of a similar age in Irish racing yards [15] but is considered to afford clinical protection from field viruses closely related to the virus in the vaccine [20]. The horses in the current study had commenced 
their primary course of two doses of vaccine one month apart from seven to 12 months of age and thereafter had received six-monthly boosters. The manufacturers' of Proteq Flu-TE and Duvaxyn IE-T Plus recommend annual boosters and the manufacturer of Equip recommends 12 to 15 month boosters after a primary course of three vaccinations. However, the practice of vaccinating horses, particularly sport horses, at six-monthly intervals is not uncommon. In the early 1980s, the Federation Equestre Internationale (FEI), the international governing body of equestrian sports, introduced a mandatory primary vaccination schedule of three doses of vaccine followed by annual revaccination. However, in 2004, the FEI approved a rule change requiring all horses competing in FEI competitions to be vaccinated within 6 months and 21 days of competing. This was in response to several reports that more frequent vaccination significantly reduced the risk of influenza outbreaks [21-23]. Vaccination breakdown has been recorded as occurring primarily, but not exclusively, among young horses which had not received a booster vaccination within the previous six months [4].

In this study, transient fever and injection site reactions were observed in several horses vaccinated with each vaccine. Such adverse reactions are documented by the vaccine manufacturers and, although one manufacturer suggests that these reactions are rare, no significant difference was found between the three vaccines in this study. A prominent acute phase response to vaccination with equine influenza vaccines has been documented [24]. Horses respond not only to the viral component of a vaccine but also to non-target antigens such as proteins derived from the embryonated eggs or cells in which the virus is propagated, and vaccine excipients. This response varies with the individual and may increase with repeat vaccination [25]. For human athletes, it is recommended that vaccinations should be scheduled in a way that possible side effects are least likely to occur in periods of competition [26]. The FEI allow a 21-day grace period to allow for strategic vaccination scheduling. This should not jeopardise the immune status, as results of this study provide evidence that the decline in antibody titre induced by three different vaccine types within the grace period is minimal.

The horses responded well to booster vaccination with the vaccines included in the study. In a previous study, eighteen of 44 National Hunt race horses (41\%) did not experience a significant increase in titre after receiving their annual booster [14] but only two of the sport horses in this study failed to demonstrate an increase of $\geq 25 \mathrm{~mm}^{2}$ post booster vaccination. The delayed response to the recombinant vaccine compared to the whole-inactivated and subunit vaccines in the current study corroborates the findings in racehorses and provides further support for the recommendation that Proteq Flu-TE be administered no later than two weeks prior to an event [27]. FEI rules permit administration of an equine influenza vaccine up to seven days before arrival at the event, which may not be adequate to provide the best protection afforded by certain vaccines.

The pattern of humoral antibody response was similar for the three vaccines and to that observed previously in racehorses [14]. The antibodies peaked between two and three weeks post vaccination and decreased sharply by three months post vaccination. In the previous study in racehorses, there was no significant difference observed between antibody response induced by any of six commercial vaccines including ProteqFlu-TE, Equip FT and Duvaxyn IE-T-Plus. However, in the current study, Tukey's HSD indicated that on the basis of antibody titres, Equip FT and Duvaxyn IE-T-Plus were superior to ProteqFlu-TE up to three months post vaccination. Thereafter, there was no significant difference and the antibody titre induced by the three vaccines declined gradually to levels associated with clinical protection rather than virological protection.

The three vaccines used in this study represent a diversity of technologies and have proved to be efficacious in the reduction in clinical signs and virus shedding in experimental challenge studies [6,7,28-32]. All three have been shown to elicit both a humoral and cell mediated response [13,14,30-32]. Many factors can affect the efficacy of an equine influenza vaccine including the relatedness of the virus strains in the vaccine to circulating virus. It has been demonstrated in the field that horses require higher antibody titres for protection if there is a mismatch between the circulating virus and the vaccine strains [8]. The recombinant vaccine ProteqFlu-TE did not induce the same level of humoral antibody response as Duvaxyn IE-T Plus and Equip TE, but was the only vaccine in this study to be updated in line 
with the OIE recommendations for equine influenza vaccine strain composition [33], suggesting that its protection threshold may be lower than vaccines with older strains. Furthermore, it is important to consider that this is a second-generation live recombinant vaccine which presents the influenza HA to the host in a manner that is considered to mimic natural infection and stimulate a complex immune response. However, the measurement of cellular or mucosal immunity was beyond the scope of this study and the most reliable evaluation of vaccine efficacy can only take place in the field when regularly vaccinated horses are exposed to virus during an outbreak of influenza.

\section{Conclusions}

Transient fever and injection site reactions were observed in several horses vaccinated with the subunit, whole-inactivated and recombinant vaccines but there was no significant difference in reactogenicity to the different formulations. All three vaccines elicited a significant antibody response but AUC analysis revealed a significantly lower response for the subunit and canarypox vaccines compared to the whole inactivated vaccine. There was a delayed response to the recombinant vaccine and for up to three months post vaccination the antibody titres were lower than those elicited in response to vaccination with the whole-inactivated and subunit vaccines. From three to six months post vaccination there was no significant difference in vaccine performance.

Supplementary Materials: The following are available online at http://www.mdpi.com/2076-393X/8/3/372/s1, Table S1: SRH antibody titres (area of haemolysis, $\mathrm{mm}^{2}$ ) against A/equine/Meath/1/2007 and A/equine/South Africa/4/2003, and mean H3N8 antibody titres calculated for individual horses.

Author Contributions: Conceptualisation, A.C. and M.V.; methodology, A.C., J.G., C.W. and M.V.; formal analysis, A.C., C.W., J.G., J.E. and M.G.; investigation, J.E. and J.G.; resources, A.C. and M.V.; data curation, J.E., J.G. and C.W.; writing-original draft preparation, A.C., J.E. and J.G.; writing-review and editing, A.C., M.V., J.E. and M.G.; visualisation, J.E. and M.G.; supervision, A.C. and M.V.; project administration, A.C.; funding acquisition, A.C. and M.V. All authors have read and agreed to the published version of the manuscript.

Funding: The laboratory work which was carried out at the Irish Equine Centre was supported by the Irish Department of Agriculture, Food and the Marine and the World Organisation for Animal Health (OIE).

Acknowledgments: The authors wish to thank Paul Schockemoehle and the team of Lewitz Stud for their invaluable contribution to this study.

Conflicts of Interest: The authors declare no conflict of interest.

\section{References}

1. Cullinane, A.; Newton, J.R. Equine influenza-A global perspective. Vet. Microbiol. 2013, 167, 205-214. [CrossRef]

2. Paillot, R. A systematic review of recent advances in equine influenza vaccination. Vaccines 2014, 2, 797. [CrossRef]

3. Gildea, S.; Garvey, M.; Lyons, P.; Lyons, R.; Gahan, J.; Walsh, C.; Cullinane, A. Multifocal equine influenza outbreak with vaccination breakdown in thoroughbred racehorses. Pathogens 2018, 7, 43. [CrossRef]

4. Gildea, S.; Lyons, P.; Lyons, R.; Gahan, J.; Garvey, M.; Cullinane, A. Annual booster vaccination and the risk of equine influenza to Thoroughbred racehorses. Equine Vet. J. 2020, 52, 509-515. [CrossRef] [PubMed]

5. Mumford, J.A.; Wood, J. Establishing an acceptability threshold for equine influenza vaccines. Dev. Biol. Stand. $1992,79,137-146$.

6. Mumford, J.; Jessett, D.; Rollinson, E.; Hannant, D.; Draper, M. Duration of protective efficacy of equine influenza immunostimulating complex/tetanus vaccines. Vet. Rec. 1994, 134, 158-162. [CrossRef]

7. Mumford, J.A.; Jessett, D.; Dunleavy, U.; Wood, J.; Hannant, D.; Sundquist, B.; Cook, R.F. Antigenicity and immunogenicity of experimental equine influenza ISCOM vaccines. Vaccine 1994, 12, 857-863. [CrossRef]

8. Newton, J.R.; Verheyen, K.; Wood, J.L.; Yates, P.J.; Mumford, J.A. Equine influenza in the United Kingdom in 1998. Vet. Rec. 1999, 145, 449-452. [CrossRef] [PubMed]

9. Newton, J.R.; Townsend, H.G.; Wood, J.L.; Sinclair, R.; Hannant, D.; Mumford, J.A. Immunity to equine influenza: Relationship of vaccine-induced antibody in young Thoroughbred racehorses to protection against field infection with influenza A/equine-2 viruses (H3N8). Equine Vet. J. 2000, 32, 65-74. [CrossRef] 
10. Mumford, J.A. Collaborative study for the establishment of three European Pharmacopoeia Biological Reference Preparations for equine influenza horse antiserum. Pharmeuropa 2000, 1, 7-21.

11. Daly, J.; Daas, A.; Behr-Gross, M.E. Collaborative study for the establishment of a candidate equine influenza subtype 2 American-like strain A/EQ/South Africa/4/03-horse antiserum biological reference preparation. Pharmeuropa Bio/Biol. Stand. Programme EDQM 2007, 2007, 7-14.

12. Mumford, J.A. Epidemiology and vaccinology of equine influenza, quality control of equine influenza vaccines. In Proceedings of the International Symposium Organised by the European Directorate for the Quality of Medicines (EDQM), Council of Europe, Budapest, Hungary, 10-11 December 2001; EDQM: Strasbourg, France, 2001; pp. 7-12.

13. Gildea, S.; Arkins, S.; Walsh, C.; Cullinane, A. A comparison of antibody responses to commercial equine influenza vaccines following primary vaccination of Thoroughbred weanlings-A randomised blind study. Vaccine 2011, 29, 9214-9223. [CrossRef] [PubMed]

14. Gildea, S.; Arkins, S.; Walsh, C.; Cullinane, A. A comparison of antibody responses to commercial equine influenza vaccines following annual booster vaccination of National Hunt horses-A randomised blind study. Vaccine 2011, 29, 3917-3922. [CrossRef] [PubMed]

15. Gildea, S.; Arkins, S.; Cullinane, A. A comparative antibody study of the potential susceptibility of Thoroughbred and non-Thoroughbred horse populations in Ireland to equine influenza virus. Influenza Other Respir. Viruses 2010, 4, 363-372. [CrossRef] [PubMed]

16. Gildea, S.; Quinlivan, M.; Arkins, S.; Cullinane, A. The molecular epidemiology of equine influenza in Ireland from 2007-2010 and its international significance. Equine Vet. J. 2012, 44, 387-392. [CrossRef] [PubMed]

17. R CoreTeam. R: A Language and Environment for Statistical Computing; R Foundation for Statistical Computing: Vienna, Austria, 2016.

18. Heldens, J.G.M.; Weststrate, M.W.; van den Hoven, R. Area under the curve calculations as a tool to compare the efficacy of equine influenza vaccines-A retrospective analysis of three independent field trials. J. Immunol. Methods 2002, 264, 11-17. [CrossRef]

19. Cullinane, A.; Gahan, J.; Walsh, C.; Nemoto, M.; Entenfellner, J.; Olguin-Perglione, C.; Garvey, M.; Huang Fu, T.Q.; Venner, M.; Yamanaka, T.; et al. Evaluation of current equine influenza vaccination protocols prior to shipment, guided by OIE standards. Vaccines 2020, 8, 107. [CrossRef]

20. Daly, J.M.; Yates, P.J.; Newton, J.R.; Park, A.; Henley, W.; Wood, J.L.N.; Davis-Poynter, N.; Mumford, J.A. Evidence supporting the inclusion of strains from each of the two co-circulating lineages of H3N8 equine influenza virus in vaccines. Vaccine 2004, 22, 4101-4109. [CrossRef]

21. de la Rua-Domenech, R.; Reid, S.W.; Gonzalez-Zariquiey, A.E.; Wood, J.L.; Gettinby, G. Modelling the spread of a viral infection in equine populations managed in Thoroughbred racehorse training yards. Prev. Vet. Med. 1999, 47, 61-77. [CrossRef]

22. Park, A.W.; Wood, J.L.; Newton, J.R.; Daly, J.; Mumford, J.A.; Grenfell, B.T. Optimising vaccination strategies in equine influenza. Vaccine 2003, 21, 2862-2870. [CrossRef]

23. Newton, J.R.; Park, A.W.; Wood, J.L.N. Maximizing the benefits of vaccination against equine influenza. In Equine Respiratory Diseases; Lekeux, P., Ed.; International Veterinary Information Service: Ithaca, NY, USA, 2004.

24. Andersen, S.A.; Petersen, H.H.; Ersbøll, A.K.; Falk-Rønne, J.; Jacobsen, S. Vaccination elicits a prominent acute phase response in horses. Vet. J. 2012, 191, 199-202. [CrossRef] [PubMed]

25. Gershwin, L.J.; Netherwood, K.A.; Norris, M.S.; Behrens, N.E.; Shao, M.X. Equine IgE responses to non-viral vaccine components. Vaccine 2012, 30, 7615-7620. [CrossRef] [PubMed]

26. Gärtner, B.C.; Meyer, T. Vaccination in elite athletes. Sports Med. 2014, 44, 1361-1376. [CrossRef]

27. Minke, J.M.; Toulemonde, C.E.; Coupier, H.; Guigal, P.M.; Dinic, S.; Sindle, T.; Jessett, D.; Black, L.; Bublot, M.; Pardo, M.C.; et al. Efficacy of a canarypox-vectored recombinant vaccine expressing the hemagglutinin gene of equine influenza H3N8 virus in the protection of ponies from viral challenge. Am. J. Vet. Res. 2007, 68, 213-219. [CrossRef]

28. Crouch, C.F.; Daly, J.; Hannant, D.; Wilkins, J.; Francis, M.J. Immune responses and protective efficacy in ponies immunised with an equine influenza ISCOM vaccine containing an 'American lineage' H3N8 virus. Vaccine 2004, 23, 418-425. [CrossRef] [PubMed] 
29. Bryant, N.A.; Paillot, R.; Rash, A.S.; Medcalf, E.; Montesso, F.; Ross, J.; Watson, J.; Jeggo, M.; Lewis, N.S.; Newton, J.R.; et al. Comparison of two modern vaccines and previous influenza infection against challenge with an equine influenza virus from the Australian 2007 outbreak. Vet. Res. 2010, 41, 19. [CrossRef] [PubMed]

30. Paillot, R.; Kydd, J.H.; Sindle, T.; Hannant, D.; Edlund Toulemonde, C.; Audonnet, J.C.; Minke, J.M.; Daly, J.M. Antibody and IFN-gamma responses induced by a recombinant canarypox vaccine and challenge infection with equine influenza virus. Vet. Immunol. Immunopathol. 2006, 112, 225-233. [CrossRef] [PubMed]

31. Paillot, R.; Grimmett, H.; Elton, D.; Daly, J.M. Protection, systemic IFNgamma, and antibody responses induced by an ISCOM-based vaccine against a recent equine influenza virus in its natural host. Vet. Res. 2008, 39, 21. [CrossRef]

32. Paillot, R.; Prowse, L.; Montesso, F.; Huang, C.M.; Barnes, H.; Escala, J. Whole inactivated equine influenza vaccine: Efficacy against a representative clade 2 equine influenza virus, IFNgamma synthesis and duration of humoral immunity. Vet. Microbiol. 2013, 162, 396-407. [CrossRef]

33. OIE. Expert surveillance panel on equine influenza vaccine composition-Conclusions and recommendations. Off. Int. Epizoot. Bull. 2010, 2, 44-45.

(C) 2020 by the authors. Licensee MDPI, Basel, Switzerland. This article is an open access article distributed under the terms and conditions of the Creative Commons Attribution (CC BY) license (http://creativecommons.org/licenses/by/4.0/). 